\title{
Las colecciones y servicios de las bibliotecas digitales: criterios para su evaluación
}

\author{
Digital libraries collections and services: evaluation criteria
}

\author{
Miguel Ángel Amaya Ramírez \\ Facultad de Filosofía y Letras, Universidad Nacional Autónoma de México, \\ Ciudad Universitaria, México, D.F. C.P. 04510
}

\begin{abstract}
Resumen
Se presentan y se analizan los principales criterios para evaluar las colecciones y los servicios de las bibliotecas digitales, tomando como base los trabajos de diferentes autores que han investigado sobre la obtención de dichos criterios. Se fundamenta que los servicios y las colecciones pueden ser evaluados desde diferentes niveles, por lo que se seleccionaron los mejores criterios, basándose en los siete niveles de evaluación que propone Tefko Saracevic: social, institucional, individual, interfase, ingeniería, procesamiento y contenido. Entre las reflexiones finales, encontramos que la existencia de diferentes tipos de criterios condiciona la forma en la que se evalúan las colecciones y los servicios de las denominadas bibliotecas digitales. Asimismo, se puede decir que este tipo de bibliotecas son entes que contienen una gran cantidad de elementos, los cuales pueden ser susceptibles de evaluar, aunque no existen muchos documentos que hayan tratado el tema de la evaluación de este tipo de unidades de información.
\end{abstract}

Palabras clave: Bibliotecas digitales. Colecciones digitales . Criterios de evaluación.

\section{Introducción}

El avance tecnológico que se ha desarrollado en la última década y la proliferación y producción de diferentes tipos de documentos electrónicos ha permitido que los servicios bibliotecarios se expandan, utilizando esta tecnología.

Asimismo permiten que se puedan compartir recursos, dando paso a la creación de bibliotecas digitales, principalmente por instituciones de educación superior como universidades y centros de investigación en casi todas las áreas del conocimiento.

En la actualidad encontramos disponibles en la world wide web una gran cantidad de bibliotecas digitales, las cuales ponen a disposición de sus usuarios y a otros usuarios que navegan en la red, las colecciones digitales que los propios integrantes de su comunidad crean o servicios

\begin{abstract}
Literature review of the main evaluation criteria for digital library collections and services. It is sustained that digital library collections and services can be evaluated from different levels, hence the best criteria were selected, based upon the seven evaluation levels proposed by Tefko Saracevic: social, institutional, individual, interface, engineering, processing, and content. Among the final considerations, we found out that the existence of different types of criteria conditions the way in which to evaluate the digital library collections and services. Also, it could be said that these kind of libraries are entities containing a great quantity of elements, which could be susceptible of evaluation and, despite there are not many documents treating the evaluation of this kind of information units, some exist which do focus on creating some evaluation criteria; criteria which in some cases has been obtained from the research made in traditional libraries.
\end{abstract}

Keywords: Digital libraries. Digital collections. Evaluation criteria.

de información con los cuales tienen convenio con proveedores o editoriales. La facilidad de manejo y la gran popularidad que ha tenido la web, permite que cualquier usuario que tenga una computadora conectada a Internet pueda acceder a ella y utilizar estos recursos que en algunos casos son de acceso gratuito.

Así pues encontramos que estas bibliotecas cuentan con una gran variedad de información en sus colecciones, en diferentes formatos como: CD-ROMs, páginas web, revistas, libros y tesis electrónicas, etc.; y que se encuentran diseñadas para ser utilizadas de una manera ágil y sencilla.

Por lo anteriormente señalado, podríamos pensar que estas bibliotecas digitales son sumamente consultadas por un gran número de usuarios, de esta manera se estaría justificando la creación, desarrollo y mantenimiento de estas 
unidades de información, puesto que la biblioteca digital como tal es sumamente cara, debido a que tiene que contar con elementos como infraestructura, telecomunicaciones, colecciones y su desarrollo requiere de personal.

Por esta razón, es indispensable contar con una herramienta que nos permita conocer si verdaderamente la biblioteca digital esta siendo usada con una cierta frecuencia y si sus servicios y colecciones satisfacen las necesidades de información de sus usuarios.

Esta herramienta, por supuesto, es la evaluación. Ella nos permite comparar los objetivos de la biblioteca con el uso y acceso a la información de ésta. Así, el presente documento ésta enfocado a conocer, analizar y obtener los mejores criterios para evaluar las colecciones y servicios desde la perspectiva de los siete niveles de evaluación de bibliotecas digitales que propone Tefko Saracevic.

\section{Evaluación en la biblioteca digital}

Existen una gran cantidad de aspectos que pueden ser susceptibles de evaluarse en el ambiente de las bibliotecas digitales. Asimismo existen una serie de niveles en los cuales podemos ubicar la evaluación:

Tefko Saracevic (2000) menciona que existen siete niveles de evaluación que se pueden utilizar para evaluar aspectos de las bibliotecas digitales, divididos en dos grandes rubros; Los niveles enfocados a los usuarios y los niveles enfocados al sistema de cómputo.

Con respecto a los niveles que tratan sobre los usuarios encontramos los siguientes elementos principales: 1) nivel social, 2) nivel institucional, 3) nivel individual, y 4) nivel de interfase.

Con respecto a los niveles enfocados a los sistemas de cómputo encontramos los siguientes tres elementos primordiales: 1) nivel de ingeniería, 2) nivel de procesamiento, y 3) nivel de Contenido.

\section{Criterios para evaluar las bibliotecas digitales}

La biblioteca digital no pretende "copiar" la producción impresa, sino que debe generar una nueva estructura de la información: El uso de documentos hipertextuales hacen que un "libro electrónico" ya no sea lineal, como ocurre con la confección en papel, sino que el libro ahora también posee "profundidad". El usuario tiene acceso a la información de formas muy variadas, la biblioteca digital cuenta con la posibilidad de proveer vínculos no solo a documentos textuales, sino también a imagen y vídeo, de modo instantáneo, lo cual permite explicar dichos contenidos de formas diversas.

Todo este cambio no se puede llevar a cabo sin que se produzca un proceso de innovación tecnológica que involucra a las diferentes partes implicadas en el desarrollo de la biblioteca digital, como son la modificación de la organización de la información, su producción y la difusión de la misma

Después de haber realizado una revisión de los criterios que presentan varios autores sobre el tema de las evaluaciones de las colecciones y servicios en las bibliotecas digitales, tales como los de Wilkinson, Oliver y Bennett, a continuación se presentan los criterios de diferentes autores, entre ellos los de:

- Criterios de Caywood: acceso, diseño, contenido.

- Criterios de Ciolek: originalidad de la información, facilidad de encontrarla en la red, facilidad de acceso, estructura y organización, formato y presentación, utilidad e integridad de la información, facilidad de mantenimiento del recurso.

- Criterios de Tillman: facilidad para entender el alcance del recurso, facilidad para identificar el meta-dato, estabilidad de la información, facilidad de uso.

- Criterios de Smith: alcance, contenido, diseño gráfico y multimedia, propósito, revisiones, aplicabilidad, costo.

- Criterios de Grassian: contenido y evaluación, fuente y fecha, estructura, otras cuestiones

- Criterios de Harris: credibilidad, exactitud, razonabilidad, legitimidad de la información.

- Criterios de Cohen: audiencia esperada, autoridad, contenido, estilo.

- Criterios de Auer: acceso, contenido, diseño, ética.

- Criterios de Codina: contenido, autoría, ergonomía, representación de la información.

Como se puede observar existen una gran cantidad de criterios que se pueden utilizar para evaluar las colecciones que integran las bibliotecas digitales y que se tienen que combinar entre ellos para poder conocer si las colecciones y la forma de acceder a ellas están cumpliendo cabalmente los objetivos para lo que fueron diseñados y adquiridos en la biblioteca digital, es decir, satisfacer las necesidades de información de los usuarios. 
Por otro lado, existen muchos criterios que se pueden utilizar para evaluar los servicios bibliotecarios en las bibliotecas digitales. William E. Moen y Kathleen (2002) proponen los siguientes: servicio de búsqueda y recuperación de recursos académicos en internet, servicio de acceso, servicio de consulta electrónica o econsulta, servicio de instrucción o educación de usuarios, servicio de cuenta al usuario

A partir de estos servicios se presentan los diferentes criterios que existen para evaluarlos, entre ellos podemos mencionar los siguientes: disponibilidad, recuperación de información, autenticidad, exhastividad, pertinencia, utilización, protección de la propiedad intelectual, autenticidad, relevancia, calidad, adecuación a las necesidades de los usuarios, precisión de la búsqueda, tiempo de respuesta, y esfuerzo del usuario, integridad en la investigación (índice de factor de utilidad)

Como se puede observar, en esta selección de criterios para evaluar los servicios y las colecciones de las bibliotecas digitales, existe una cantidad de ellos que nos pueden ayudar a llevar a cabo una evaluación, todo depende del nivel en el que queramos enfocar nuestra evaluación, que puede ser desde el punto de vista de los usuarios o del sistema de cómputo.

\section{Análisis de los criterios para evaluar las colecciones de la biblioteca digital}

Después de haber revisado minuciosamente los diferentes criterios que existen para evaluar las colecciones y los servicios en las bibliotecas digitales y haber seleccionado los mejores de acuerdo a nuestra opinión, es necesario que se haga un breve análisis de ellos para poder ubicarlos en el nivel de evaluación que les corresponde. Este análisis consiste en describir cada uno de los diferentes criterios de evaluación antes señalados, desde el punto de vista del objetivo que persigue y así tratar de ubicar cada uno de ellos en los siete niveles de evaluación de Tefko Saracevic.

Acceso: El acceso es muy importante puesto que, como ya se ha mencionado, las bibliotecas digitales no se encuentran físicamente en un lugar, sino en un ambiente virtual accesible por Internet, lo que permite que cualquier persona que tenga una computadora conectada en red pueda entrar al sitio o URL y tratar de consultar la información que se encuentra disponible en la biblioteca. Por esta razón, las bibliotecas digitales restringen el acceso total o parcial a sus materiales a usuarios que no son identificados como miembros de la institución. En algunos casos, el acceso a las bibliotecas digitales es solo para los usuarios que pertenecen a la institución que genera la biblioteca y que se encuentran registrados o se les ha asignado un $\operatorname{login}$ y un password. En ocasiones, el acceso a la biblioteca se da únicamente utilizando computadoras que tienen una dirección IP perteneciente a la institución.

Diseño: El criterio de diseño tiene un gran valor, puesto que es aquí precisamente en donde se tienen que desarrollar y diseñar las redes y los sistemas que van a permitir el funcionamiento de la biblioteca digital, y a los que solamente los administradores de la biblioteca pueden tener acceso.

Contenido: El contenido de un recurso de información es muy importante para saber si los materiales que se están incluyendo en la biblioteca digital contienen información relevante sobre los tópicos que se estudian en la institución a la cual pertenece la biblioteca digital.

Alcance: El alcance permite conocer la especialización de un recurso de información, esto es, la profundidad con que se trata un tema, desde el punto de vista del autor, ya sea de manera general o muy específica.

Autoridad: La autoridad de un recurso, nos permite saber si el autor es reconocido en el tema que trata y si es respaldado por alguna institución con un soporte académico como una universidad, una escuela, un colegio etc. Asimismo, permite tener la seguridad de que el recurso es confiable y que puede ser utilizado por los usuarios con toda confianza para satisfacer sus necesidades de información.

Propósito: El propósito como criterio para evaluar las colecciones de la biblioteca digital nos permite saber si los documentos que formaran parte del acervo de ésta cubren los objetivos de la institución o de los programas de estudio que se verán reflejados en el uso y aprovechamiento de la comunidad académica a la que atiende la biblioteca.

Audiencia esperada: Tiene que ver principalmente con la cantidad de veces que un recurso será utilizado por sus usuarios, lo que es útil para valorar tanto el costo económico como en tiempo y espacio en el servidor. En otras palabras, si el recurso es muy utilizado por los usuarios, no importa el costo del mismo ni el espacio que utilice.

Facilidad de encontrar la información en la red: Este criterio tiene que ver un poco con las habilidades informativas de los usuarios. Esto puede parecer algo contradictorio, si estamos hablando de bibliotecas digitales, porque se supone que la información que consultan los usuarios ya ha 
sido encontrada, evaluada y organizada por los bibliotecarios y se encuentra a disposición de los usuarios en el sitio de la biblioteca. Sin embargo, en ocasiones existen sitios que no son artículos ni documentos, sino bases de datos que pueden ser gratuitas o por suscripción y el usuario debe realizar por sí solo la búsqueda de información.

Utilidad e integridad de la información: Desde el punto de vista de los usuarios, es necesario saber en que medida un recurso de información es utilizado por los usuarios, o si este mismo recurso se encuentra integrado en la base de datos pero ya no esta disponible para su consulta, tal vez porque el recurso es gratuito y el servidor en el que se encuentra ha sido dado de baja o, si es un recurso por suscripción, probablemente ya no se haya renovado el contrato.

Estabilidad de la información: Permite conocer la estructura de la base de datos en la que se encuentra almacenada la información de la biblioteca digital, principalmente si los documentos están catalogados y clasificados, y, de ser así, qué tipo de metadatos es el mejor para no perder la información en la migración de datos, pensando en que la mayoría de las bases de datos en algún momento tienen que cambiar de manejador de base de datos. Es un elemento indispensable para el procesamiento, puesto que algunas colecciones que se van a integrar a la bases de datos de la biblioteca, pueden estar en peligro de desaparecer de la red, en el caso de que sean recursos gratuitos o bien si son por suscripción se debe tener la seguridad de que estarán un buen tiempo dentro de la base de datos o por lo menos estar al pendiente de cuando vence la suscripción del recurso.

Costo: Con respecto a las colecciones o servicios de la biblioteca digital, el criterio de costo tiene un significado especial, debe equilibrarse con la necesidad de satisfacer las necesidades de información de la comunidad a la que sirve la biblioteca digital.

Facilidad de mantenimiento del recurso: Otro criterio esencial es el de la facilidad de mantenimiento del recurso, esto principalmente porque permite saber si el administrador o administradores de la biblioteca digital no tendrán problemas para dar mantenimiento a los sistemas o redes de información que almacenarán a los recursos de información que conforman la biblioteca, ya sean páginas web, documentos en diferentes formatos como pdf, word, ppt, así como bases de datos, ya sean por suscripción o gratuitas.

Originalidad: En el nivel de procesamiento es muy importante la originalidad de la información, puesto que existen en la red documentos que no son gratuitos y que pueden ser utilizados en las bibliotecas digitales, por lo tanto es necesario que se tengan el permiso o la autorización para poder utilizar el recurso sin incurrir en algún problema legal.

Facilidad para identificar el metadato: Es recomendable que las personas que trabajan en el procesamiento de los datos, puedan conocer e identificar cual estructura de meta datos es la que se esta utilizando para organizar los materiales de la biblioteca digital.

Credibilidad: Permite conocer el grado de confianza que se tiene sobre el recurso que integra la colección principalmente por los orígenes del documento o su procedencia.

Legitimidad de la información: Es necesario que el administrador de la biblioteca conozca la procedencia del recurso para evitar problemas legales, porque en la red existen recursos que no son gratuitos y que pudieron ser obtenidos sin el consentimiento del productor y esto podría traer como resultado que se este utilizando un recurso ilegal en la biblioteca.

Ética: Básicamente se enfoca a saber si las personas que hicieron la selección, la adquisición y la organización de las colecciones que se encuentran en la biblioteca digital cumplieron cabalmente con los principios morales del contenido del recurso y la legalidad de la información.

\section{Análisis de los criterios para evaluar los servicios de la biblioteca digital}

A continuación se presenta el análisis de los principales criterios de evaluación para los servicios de las bibliotecas digitales:

Disponibilidad: Nos permite conocer el nivel en el que se encuentran trabajando los servicios de la biblioteca digital, en este sentido es necesario verificar que todos los usuarios o la mayoría de ellos puedan acceder a los servicios sin ningún problema y, de ser así, el grado en el satisfacen sus necesidades de información.

Recuperación de información: En las bibliotecas digitales es vital, sobre todo porque se supone que este tipo de unidades de información están diseñadas para que los usuarios puedan recuperar la información de una manera rápida, sencilla y con la seguridad de que la información que localicen es confiable.

Exhaustividad: Se refiere a la forma en que un usuario busca información en la biblioteca, es decir las habilidades informativas con respecto a la recuperación de información, principalmente a la cantidad de información que recuperan por si 
solos o con la ayuda del personal de la biblioteca digital.

Pertinencia: Con respecto a la pertinencia de la información que se encuentra en la biblioteca, es necesario verificar que los materiales que integran los acervos de la biblioteca cubran todas las áreas de la institución y satisfaciendo así las necesidades de información de su comunidad.

Autenticidad: Se refiere al tipo de servicios que ofrece la biblioteca digital, es decir, si los servicios son atractivos para los usuarios o si son una copia de los que ofrece la biblioteca tradicional, en este sentido el usuario no ve diferencia entre ellos y puede llegar a no utilizarlos por pensar que es la misma información que recuperara en los dos tipos de biblioteca y talvez prefiera los servicios presénciales.

Utilización: La utilización de la información por parte de los usuarios de la biblioteca digital da un panorama más amplio, con respecto a los recursos de información que se incluyen en ella, puesto que un recurso con un alto nivel académico y un autor ampliamente reconocido y que además es respaldado por una institución académica, puede ser poco utilizado, asimismo un documento con un contenido muy general y elaborado por un autor no tan reconocido puede ser más consultado que el anterior.

Adecuación a las necesidades del usuario: Nace de los estudios que se realizan antes de iniciar un servicio, o sea de los estudios de usuarios en los cuales se detectan las necesidades de información de los usuarios, lo que permite saber si un servicio será utilizado por los usuarios y si satisfacerá sus necesidades de información.

Precisión en la búsqueda: Importa también en las búsquedas delegadas, es decir, en las que el usuario hace uso de los servicios de recuperación de información y solicita la ayuda del personal de la biblioteca digital. En este caso, este criterio ayuda a conocer el grado de especialización del personal al responder a la solicitud con documentos que satisfagan las necesidades de información del usuario.

Exhaustividad: Es lograr un barrido completo sobre un tema de los registros que se encuentran en la biblioteca digital y en ocasiones fuera de ella.

Pertinencia: Se puede entender como la recuperación de información adecuada para los intereses de un usuario, es decir que la información que se recupera sobre un tema tenga que ver con la necesidad de información de los usuarios.
Relevancia: El criterio de relevancia lo da únicamente el usuario, que es el que decide si los servicios y la información que encuentra en la biblioteca digital son relevantes para satisfacer sus necesidades de información.

\section{Discusión de los resultados}

Después de haber realizado un breve análisis de los principales criterios para evaluar las colecciones y los servicios de las bibliotecas digitales, a continuación se presentan los resultados de este análisis ubicando los criterios dentro del nivel que les corresponde de acuerdo a los diferentes enfoques, ya sea desde el punto de vista de los usuarios o de la clasificación de niveles de evaluación de Saracevic.

\subsection{Niveles enfocados a los usuarios.}

Nivel Social: El objetivo de este nivel es determinar si las colecciones y los servicios que integran una biblioteca digital esta ayudando a satisfacer las necesidades de información de la comunidad que la creo. Por lo tanto se pueden utilizar los siguientes criterios para evaluarlos y determinar si cumplen con el objetivo de dicho nivel.

\begin{tabular}{ll}
\hline Criterios de colecciones & Criterios de servicios \\
\hline Contenido & Disponibilidad \\
\hline Alcance & $\begin{array}{l}\text { Recuperación de } \\
\text { información }\end{array}$ \\
\hline Autoridad & Exhaustividad \\
\hline & Pertinencia \\
\hline
\end{tabular}

Nivel Institucional: El objetivo de este nivel es conocer si la temática de las colecciones que conforman el acervo de la biblioteca digital, además de los servicios, están orientadas para satisfacer las necesidades de información de la comunidad a la que se pretende servir, apoyando así los objetivos institucionales de una organización. Los criterios que se pueden utilizar para evaluarlos en este nivel son:

\begin{tabular}{ll}
\hline Criterios de colecciones & Criterios de servicios \\
\hline Propósito & Autenticidad \\
\hline Audiencia esperada & Utilización \\
\hline
\end{tabular}

Nivel Individual: El objetivo de este nivel es conocer cómo la biblioteca digital ayuda a satisfacer las necesidades de información de un usuario o de un grupo de ellos, pero no únicamente desde el punto de vista de la información como 
tal, sino también desde el aspecto de la facilidad con la que accede a estos recursos, utilizando los servicios. Los criterios que se pueden utilizar para evaluarlos son:

\begin{tabular}{ll}
\hline Criterios de colecciones & Criterios de servicios \\
\hline Contenido & $\begin{array}{l}\text { Adecuación a las } \\
\text { necesidades } \\
\text { delUsuario }\end{array}$ \\
\hline Alcance & $\begin{array}{l}\text { Precisión de la } \\
\text { búsqueda }\end{array}$ \\
\hline Autoridad & Exhaustividad \\
\hline Acceso & Pertinencia \\
\hline & Relevancia \\
\hline
\end{tabular}

Nivel Interfase: el objetivo de este nivel es comprobar la interacción de la biblioteca digital con el usuario, en el sentido del acceso, la navegación en el sitio y la búsqueda de información. Además en este nivel se puede considerar que es el enlace entre los niveles que tratan sobre los usuarios y los que tratan sobre los sistemas de cómputo. Los criterios que se pueden utilizar son:

\begin{tabular}{ll}
\hline Criterios de colecciones & Criterios de servicios \\
\hline Acceso & Esfuerzo del usuario \\
\hline Diseño & $\begin{array}{l}\text { Precisión de la } \\
\text { búsqueda }\end{array}$ \\
\hline $\begin{array}{l}\text { Facilidad de encontrarla } \\
\text { en la red }\end{array}$ & \\
\hline $\begin{array}{l}\text { Utilidad e integridad de la } \\
\text { información }\end{array}$ & \\
\hline
\end{tabular}

\subsection{Niveles enfocados a los sistemas}

Nivel de ingeniería: El objetivo de este nivel es configurar el hardware o programas con las redes de trabajo, que permitirán que los recursos de información que conforman el acervo de la biblioteca no tengan problema con respecto a su uso por parte de los usuarios. Los criterios que se pueden utilizar son:

\begin{tabular}{ll}
\hline Criterios de colecciones & Criterios de servicios \\
\hline Diseño & $\begin{array}{l}\text { Protección de la } \\
\text { propiedad intelectual }\end{array}$ \\
\hline $\begin{array}{l}\text { Estabilidad de la } \\
\text { información }\end{array}$ & Tiempo de respuesta \\
\hline Costo & \\
\hline $\begin{array}{l}\text { Facilidad del } \\
\text { mantenimiento del } \\
\text { recurso }\end{array}$ & \\
\hline
\end{tabular}

Nivel de procesamiento: El objetivo de este nivel es el de realizar los procedimientos, técnicas, algoritmos, y operaciones del sistema. Los criterios que se pueden utilizar son:

\begin{tabular}{ll}
\hline Criterios de colecciones & Criterios de servicios \\
\hline $\begin{array}{l}\text { Originalidad de la } \\
\text { información }\end{array}$ & Tiempo de respuesta \\
\hline $\begin{array}{l}\text { Facilidad para identificar } \\
\text { el metadato }\end{array}$ & $\begin{array}{l}\text { Integridad en la } \\
\text { investigación }\end{array}$ \\
\hline $\begin{array}{l}\text { Estabilidad de la } \\
\text { información }\end{array}$ & \\
\hline
\end{tabular}

Nivel de contenido: En este nivel se ven como son seleccionados, organizados, estructurados y administrada la colección y los servicios de información. Los criterios propuestos son:

\begin{tabular}{ll}
\hline Criterios de colecciones & Criterios de servicios \\
\hline Contenido & Calidad \\
\hline Credibilidad & Relevancia \\
\hline $\begin{array}{l}\text { Legitimidad de la } \\
\text { información }\end{array}$ & Autenticidad \\
\hline Autoría & Utilización \\
\hline Ética & \\
\hline
\end{tabular}

Como se puede apreciar en este apartado los criterios que nos pueden ayudar a evaluar las colecciones y los servicios de las bibliotecas digitales pueden utilizarse en distintos niveles, dependiendo de la finalidad de la evaluación.

\section{Conclusiones}

Finalmente, y a manera de conclusión, podemos mencionar que la importancia que tienen los criterios de evaluación de las colecciones y servicios es sustancial para poder realizar evaluaciones en las bibliotecas digitales.

Existen muchos trabajos sobre la evaluación de las bibliotecas digitales y sobre los criterios para evaluarlas, el problema es que al ofrecer una gran cantidad de ellos en forma de lista nos es difícil discernir los que nos pueden servir y los que no para hacer una evaluación, o cuales son los mejores.

En realidad, creo que no existen mejores o peores, simplemente es necesario saber desde que nivel queremos realizar nuestra evaluación. Los niveles que ofrece Tefko Saracevic pueden ser una buena solución, lo único que necesitamos es ubicar los diferentes criterios dentro del nivel que queremos evaluar.

\section{Referencias}

Bertot, J.C. (2004). Assessing Digital Library Services: Approaches, Issues, and Considerations. // Proceedings 
of the International Symposium on Digital Libraries and Knowledge Communities in Networked Information Society. Japan, Tsukuba, Ibaraki, March 2-5.

Codina, Lluís. (2000). Evaluación de recursos digitales en línea: conceptos, indicadores y métodos. // Revista española de documentación científica. 23:1 (2000) 22.

Moen, William E.; Murray, Kathleen. ( 2002 ). A service based approach for virtual libraries. http://www.unt.edu/ wmoen/publications/MoenVirtualLibrariesZLOTFall2002. pdf (2002-03-16)
Saracevic, Tefko. (2000). Digital Library Evaluation: Toward an Evolution of Concepts. http://www.scils.rutgers.edu/ -tefko/LibraryTrends2000.pdf (2006-04-28)

Wilkinson, GL.; Oliver, KM.; Bennett, LT. (1997). Evaluating the Quality of Internet Information Sources: quality indicators as ranked by experienced Internet users. http://itech1.coe.uga.edu/Faculty/gwilkinson/rankings.ht $\mathrm{ml}$ (2006-04-28). 